ARTICLE

\title{
Long-range ballistic transport of Brown-Zak fermions in graphene superlattices
}

Julien Barrier (1) 1,2,5, Piranavan Kumaravadivel (1) 1,2,5, Roshan Krishna Kumar ${ }^{1,2}$, L. A. Ponomarenko ${ }^{1,3}, \mathrm{Na} \mathrm{Xin}^{1,2}$, Matthew Holwill (1) 2, Ciaran Mullan (1) ', Minsoo Kim (1) 1, R. V. Gorbachev (1) 1,2, M. D. Thompson (1) ${ }^{3}$,

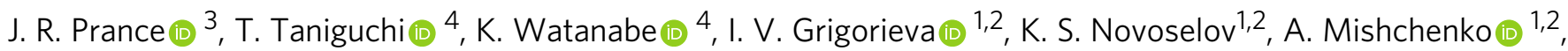
V. I. Fal'ko (1) ${ }^{1,2}$, A. K. Geim (i) ${ }^{1,2} \&$ \& A. I. Berdyugin (i) ${ }^{1,2}$

In quantizing magnetic fields, graphene superlattices exhibit a complex fractal spectrum often referred to as the Hofstadter butterfly. It can be viewed as a collection of Landau levels that arise from quantization of Brown-Zak minibands recurring at rational $(p / q)$ fractions of the magnetic flux quantum per superlattice unit cell. Here we show that, in graphene-on-boronnitride superlattices, Brown-Zak fermions can exhibit mobilities above $10^{6} \mathrm{~cm}^{2} \mathrm{~V}^{-1} \mathrm{~s}^{-1}$ and the mean free path exceeding several micrometers. The exceptional quality of our devices allows us to show that Brown-Zak minibands are $4 q$ times degenerate and all the degeneracies (spin, valley and mini-valley) can be lifted by exchange interactions below $1 \mathrm{~K}$. We also found negative bend resistance at $1 / q$ fractions for electrical probes placed as far as several micrometers apart. The latter observation highlights the fact that Brown-Zak fermions are Bloch quasiparticles propagating in high fields along straight trajectories, just like electrons in zero field.

\footnotetext{
${ }^{1}$ Department of Physics and Astronomy, University of Manchester, Manchester M13 9PL, UK. ${ }^{2}$ National Graphene Institute, University of Manchester, Manchester M13 9PL, UK. ${ }^{3}$ Department of Physics, University of Lancaster, Lancaster LA1 4YW, UK. ${ }^{4}$ National Institute for Materials Science, Ibaraki 3050044, Japan. ${ }^{5}$ These authors contributed equally: Julien Barrier, Piranavan Kumaravadivel. ${ }^{凶}$ email: geim@manchester.ac.uk; alexey.berdyugin@manchester. ac.uk
} 
$\mathrm{V}$ an der Waals assembly offers a possibility to create materials by stacking atomically-thin layers of different crystals $^{1-3}$. One of the simplest and most studied van der Waals heterostructures is graphene encapsulated between two hexagonal boron nitride (hBN) crystals. The encapsulation protects graphene from extrinsic disorder ${ }^{4,5}$, allowing ultra-high electronic quality and micrometer-scale ballistic transport often limited only by edge scattering ${ }^{6,7}$. A special case of encapsulated graphene heterostructures is graphene superlattices, where crystallographic axes of graphene and $\mathrm{hBN}$ are intentionally aligned. A small (1.8\%) mismatch between graphene and hBN crystal lattices results in a periodic moiré potential acting on charge carriers in graphene and leading to the formation of electronic minibands ${ }^{1-3,8-17}$.

A relatively large $(\sim 14 \mathrm{~nm})$ periodicity of graphene-on-hBN superlattices has also made it possible to study the regime of Hofstadter butterflies, which requires the magnetic flux $\phi$ per superlattice unit cell to be comparable to the flux quantum $\phi_{0}$ in experimentally-accessible magnetic fields $B$. At $B=B_{\mathrm{p} / \mathrm{q}}$ corresponding to $\phi=\phi_{0} p / q$, where $p$ and $q$ are integer, the translational symmetry of the electronic system is restored (despite the presence of a quantizing magnetic field) and the superlattice's electronic spectrum can again be described in terms of Bloch states $^{18-29}$, just like in $B=0$. These high-field Bloch states are characterized by their own miniband spectra ${ }^{25-29}$ different from the zero- $B$ spectrum of graphene-on-hBN superlattices. The associated quasiparticles are referred to as Brown-Zak (BZ) fermions. According to the group-theory analysis, an electronic spectrum for each realization of $\mathrm{BZ}$ fermions should have an additional $q$-fold degeneracy ${ }^{18-27,30,31}$ (that is, contains $q$ equivalent mini-valleys). This degeneracy is additional to the 4fold spin and valley degeneracy of graphene's original spectrum. Importantly, BZ fermions are Bloch quasiparticles, like electrons in solids or Dirac fermions, and, at $B=B_{\mathrm{p} / \mathrm{q}}$, they move through the superlattice as if the applied field is zero. Away from these exact values, BZ fermions experience an effective magnetic field $B_{\text {eff }}=B-B_{\mathrm{p} / \mathrm{q}}$ (refs. $\left.{ }^{18-27,30,31}\right)$.

\section{Results}

Experimental devices and measurement setup. We report the electronic properties of BZ fermions with different $p$ and $q$ using high-quality graphene superlattices. These devices were fabricated using the standard dry transfer procedures, where the studied graphene crystal was carefully aligned with one of the encapsulating $\mathrm{hBN}$ crystals using the crystallographic edges ${ }^{11}$. The alignment was verified by Raman spectroscopy 32 prior to encapsulation with the second hBN crystal. The latter was intentionally misaligned to avoid competing moiré patterns ${ }^{33-35}$. The assembled stacks were placed on an oxidized Si wafer, which allowed us to apply the back-gate voltage $V_{\mathrm{g}}$ to control the carrier density $n$. We studied six devices that were shaped into the multiterminal Hall bar geometry and had the main channel widths $W$ ranging from 2 to $17 \mu \mathrm{m}$ (see Fig. 1a and Supplementary Note 1). The devices were first characterized by measuring their longitudinal resistivity $\rho_{\mathrm{xx}}$ in zero $B$ and Hall resistivity $\rho_{\mathrm{xy}}$ in small non-quantising $B$ below $0.1 \mathrm{~T}$. The latter enabled us to find the $n\left(V_{\mathrm{g}}\right)$ dependences, except for gate voltages close to the neutrality points (NPs) and van Hove singularities (vHS), where $\rho_{\mathrm{xy}}$ reversed its sign and could no longer be described by the standard dependence $\rho_{\mathrm{xy}}=B / n e$ ( $e$ is the electron charge). All our devices exhibited very high carrier mobilities $\mu=\left(\rho_{\mathrm{xx}} n e\right)^{-1}$ of the order of $10^{6} \mathrm{~cm}^{2} \mathrm{~V}^{-1} \mathrm{~s}^{-1}$, which were still somewhat reduced by edge scattering because of finite $W$ (Fig. 1b). We corroborated the high quality of our devices using transverse magnetic focusing measurements (Supplementary
Note 2). In the main text, we focus on two large-width devices (D1 and D2) exhibiting highest $\mu$.

Figure 1c shows a map of the longitudinal conductivity $\sigma_{\mathrm{xx}}=$ $\rho_{\mathrm{xx}} /\left(\rho_{\mathrm{xx}}^{2}+\rho_{\mathrm{xy}}^{2}\right)$ as a function of $V_{\mathrm{g}}$ and $B$ in fields up to $18 \mathrm{~T}$. The main features on such maps have been well understood in terms of the Hofstadter spectrum for Dirac fermions in moiré superlattices $^{11-17,22}$. One can see numerous Landau levels (LLs) fanning out from the main and secondary NPs (miniband edges) which are located at $V_{\mathrm{g}}$ near 0 and $\pm 45 \mathrm{~V}$, respectively. There are also pronounced funneling features near van Hove singularities at $V_{\mathrm{g}} \approx-60,-35,+40$ and $+55 \mathrm{~V}$ in both hole $(-)$ and electron $(+)$ parts of the spectrum. Another important attribute of the map is horizontal yellow streaks that occur at $\phi / \phi_{0} p / q$ (Fig. 1c). If temperature $T$ is increased above $100 \mathrm{~K}$ so that Landau quantization is strongly suppressed, the horizontal streaks become the only dominant feature on such transport maps ${ }^{28,29}$. In the high- $T$ regime, the streaks represent oscillations in both $\rho_{\mathrm{xx}}$ $(B)$ and $\rho_{\mathrm{xx}}(B)$ at a constant $V_{\mathrm{g}}$. Their $1 / B$ frequency is independent of $n$, and they were named BZ oscillations ${ }^{28}$. The horizontal streaks are seen in Fig. 1c correspond to maxima in $\sigma_{\mathrm{xx}}$ and zeros in $\rho_{\mathrm{xx}}$ and, as explained in the introduction, reflect the recovery of translational symmetry ( $p$ flux quanta penetrate through $q$ superlattice unit cells) and the emergence of Bloch

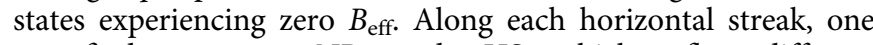
can find numerous NPs and vHS, which reflect different realizations of $\mathrm{BZ}$ fermions at each $B_{\mathrm{p} / \mathrm{q}}$. Landau mini-fans radiate from these NPs in both directions along the $B$ axis (Fig. 1c), representing Landau quantization of $\mathrm{BZ}$ electronic spectra by non-zero $B_{\text {eff }}$ (see below) ${ }^{18-29}$. Another notable feature of the shown $\sigma_{\mathrm{xx}}$ map is a repetitive triangular-like pattern seen most clearly between 2 and $12 \mathrm{~T}$, especially for positive $V_{\mathrm{g}}$. The yellow triangles are made of horizontal streaks at zero $B_{\text {eff }}$, vertical streaks emerging from NPs for BZ fermions and slanted streaks originating from vHS.

Ballistic transport. The transport properties of BZ fermions can be analyzed in the same way as in Fig. 1b for Dirac fermions. The results are plotted in Fig. 1d for the case of $\phi / \phi_{0}=1 / 2$ and show that, away from NPs and vHS, BZ fermions in our devices exhibit $\mu$ reaching a few $10^{6} \mathrm{~cm}^{2} \mathrm{~V}^{-1} \mathrm{~s}^{-1}$. This is comparable to $\mu$ of Dirac fermions in zero $B$. Another important characteristic of charge carrier transport is the mean free path $l$. It can be evaluated for both Dirac and BZ fermions, using the standard expression $\sigma_{\mathrm{xx}}=g \frac{e^{2}}{h}\left(\frac{k_{\mathrm{F}} l}{2}\right)$ where $h$ is the Planck constant, $k_{\mathrm{F}}$ is the Fermi momentum and $g$ is the degeneracy. In zero $B, g=4$ because of the spin and valley degeneracy of Dirac fermions. BZ fermions are expected to have an additional, mini-valley degeneracy ${ }^{18-27,30,31}$, which is equal to $q$ (that is, $g=8$ for the case of Fig. 1d). Using these $g$, we calculated $l$ as shown in the bottom panels of Fig. 1b, d. The mean free path in zero $B$ reached $>20$ $\mu \mathrm{m}$, implying that ballistic transport in our devices is limited by $W$ rather than impurity scattering. For some realizations of $\mathrm{BZ}$ fermions, their mean free path also exceeds $10 \mu \mathrm{m}$, marginally smaller than $l$ for Dirac fermions (cf. Fig. 1b, d). Supplementary Note 4 provides similar analysis for $\mu$ and $l$ at other values of $q$.

We corroborate the existence of ballistic BZ fermions using socalled bend resistance geometry ${ }^{5,36}$ sketched in Fig. 2a. The geometry allows one to detect if charge carriers can move ballistically over the entire channel width $W$, along straight trajectories connecting current and voltage contacts (see caption of Fig. 2a). If this is the case, ballistic transport gives rise to the negative sign of the bend resistance $R_{\mathrm{b}}$ (ref. ${ }^{36}$ ) in contrast to its conventional, positive sign for diffusive (ohmic) transport. As expected from very long $l$ of Dirac fermions, $R_{\mathrm{b}}$ was negative in 
a

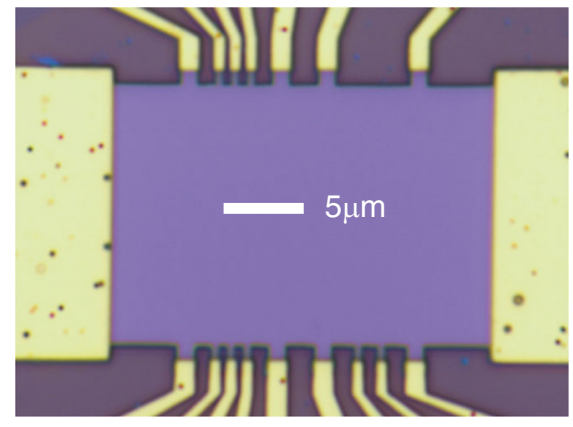

b

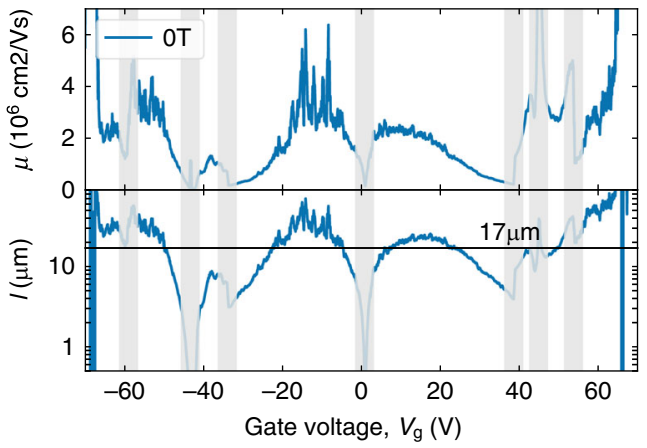

C

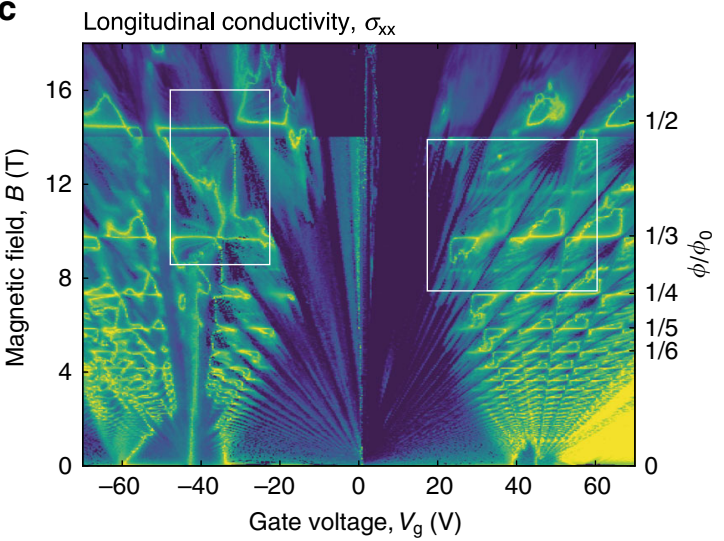

d

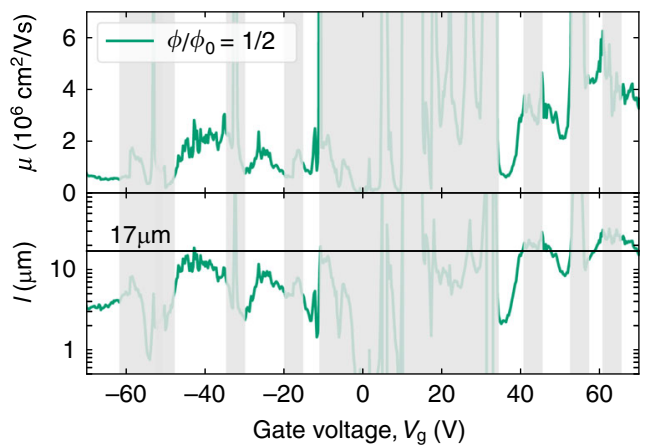

Fig. 1 High-quality graphene superlattices and their transport properties. a Optical micrograph of one of our devices (D1; twist angle $\theta$ between graphene and $\mathrm{hBN}$ of about $0.4^{\circ}$ ). The Hall bar is seen in violet with golden electrical contacts. b Mobility and mean free path for D1 measured at zero $B$ and 10 mK. Semitransparent vertical strips indicate the doping regions around NPs and vHS where $n$ could not be extracted directly from Hall measurements and charge inhomogeneity also plays a role. To calculate $\mu$ and $/$ within the shaded regions, we assumed a constant gate capacitance and linearly extrapolated the $n\left(V_{g}\right)$ dependences found sufficiently far from NPs and vHS (Supplementary Note 3). The noisy behavior at large values of $\mu$ and $/$ arises from $\rho_{\mathrm{xx}}$ becoming small ( 1Ohm, about 4 orders of magnitude smaller than that at the NPs). The horizontal black line indicates the device width $W$. $\mathbf{c} \sigma_{\mathrm{xx}}\left(V_{\mathrm{g}}, B\right)$ measured by sweeping $V_{g}$ and varying $B$ in small steps of $40 \mathrm{mT} . T=10$ and $250 \mathrm{mK}$ below and above $14 \mathrm{~T}$, respectively. Indigo-to-yellow colors: Log scale truncated between $38 \mathrm{nS}$ and $16 \mathrm{mS}$ for $B<14 \mathrm{~T}$ and between $4 \mathrm{nS}$ and $0.4 \mathrm{mS}$ above $14 \mathrm{~T}$. White rectangles: these regions are shown in finer detail in Figs. 3 and 4. d Same as in (b) but for $\phi / \phi_{0}=1 / 2(B \approx 15 T) ; T=250 \mathrm{mK}$. In addition to NPs and vHS, the gray strips also cover a wide region of the quantum Hall regime $\left(\left|V_{\mathrm{g}}\right|<20 \mathrm{~V}\right)$, which is dominated by large cyclotron gaps in the main graphene spectrum. The transport data used to calculate $\mu$ and $I$ in (b) and (d) are shown in Supplementary Note 3.

zero $B$ everywhere away from NPs and vHS (Fig. 2b), confirming further the high quality of our superlattice devices. Finite $B$ bend Dirac fermion trajectories and, as expected, $R_{\mathrm{b}}$ rapidly reversed its $\operatorname{sign}^{36}$ with increasing $B$ (inset of Fig. 2b). Remarkably, our devices exhibited negative $R_{\mathrm{b}}$ also in high $B=B_{\mathrm{p} / \mathrm{q}}$, thus revealing straight trajectories over distances of several $\mu \mathrm{m}$ (Fig. $2 \mathrm{c}$, $\mathrm{d}$ and Supplementary Note 5). For comparison, the corresponding map of $\rho_{\mathrm{xx}}$ is provided in Supplementary Note 6, which shows that the measured longitudinal resistance always remained positive. The profound negative pockets in $R_{\mathrm{b}}\left(V_{\mathrm{g}}, B\right)$ appeared only around $B$ corresponding to $\phi / \phi_{0}=1 / 2,1 / 3,1 / 4$ and $1 / 5$. This is the regime where the existence of $\mathrm{BZ}$ fermions, experiencing zero $B_{\text {eff }}$, was previously inferred ${ }^{28,29}$ from maxima in $\sigma_{\mathrm{xx}}$ and zeros in $\rho_{\mathrm{xy}}$. The possibility of ballistic transport of BZ fermions was also suggested using numerical simulations ${ }^{15}$. The observed negative $R_{b}$ prove the previous conjectures unequivocally. The negative pockets in Fig. $2 \mathrm{c}$ are located between NPs and vHS for BZ fermions, similar to the case of Dirac fermions. Away from $B=B_{\mathrm{p} / \mathrm{q}}$, non-zero $B_{\text {eff }}$ bends the $\mathrm{BZ}$ fermion trajectories and the negative signal disappeared (inset of Fig. 2d), again like in the case of other Bloch quasiparticles (electrons and Dirac fermions). Our devices exhibited long-range ballistic transport of BZ fermions only for unit fractions, $\phi_{0} / q$. For example, Fig. $2 c$ reveals a pronounced set of negative $R_{\mathrm{b}}$ pockets at $\phi / \phi_{0}=1 / 5$ but no negative signal was observed for $2 / 5$ and $3 / 5$. This behavior probably stems from lower $\mu$ of BZ fermions with $p>1$, which can be attributed to their larger effective masses ${ }^{29}$.

Lifting degeneracy in BZ minibands. The high mobility of BZ fermions resulted in their Landau quantization in small $B_{\text {eff }}<1 \mathrm{~T}$, which allowed us to find experimentally the spectral degeneracy $g$ for different $p / q$. To this end, Fig. 3a shows a high-resolution map of $\sigma_{\mathrm{xx}}$ between $\phi / \phi_{0}=1 / 2$ and $1 / 4$ (part of Fig. 1c). One can clearly see many Landau mini-fans originating from NPs for different realizations of BZ fermions. For example, there are three profound mini-fans spreading from $B \approx 9.7 \mathrm{~T}\left(\phi / \phi_{0}=1 / 3\right)$ for both negative and positive $B_{\text {eff. For clarity, the conductivity map }}$ in Fig. $3 \mathrm{a}$ is replotted in Fig. $3 \mathrm{~b}$ by tracing all well-defined minima in $\sigma_{\mathrm{xx}}$. Each minimum can be described by its integer filling factor $v$, which we calculated from the minimum's slope. This representation of the Hofstadter spectrum, where LLs are plotted as a function of $n$ or $V_{\mathrm{g}}$ rather than energy, is usually referred to as the Wannier diagram $11-17,21,22$. In the diagram of Fig. $3 b$, one can identify LLs for BZ-fermion realizations at $q=2,3,4,5,7,8,9$, and 11, and for $p=1,2,3,4$, and 5. The difference in $v$ between the nearest LLs yields directly their $g$. For example, all LLs found for $\phi / \phi_{0}=1 / 2$ were separated by $\Delta v=2$ whereas those at $\phi / \phi_{0}=$ 
a
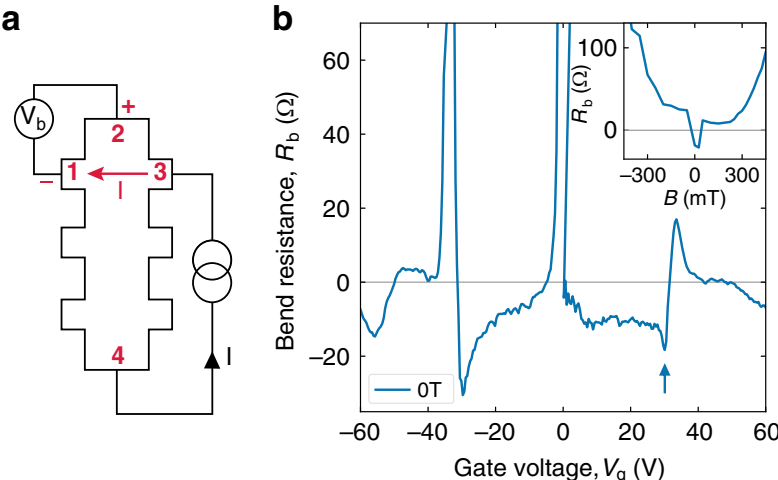

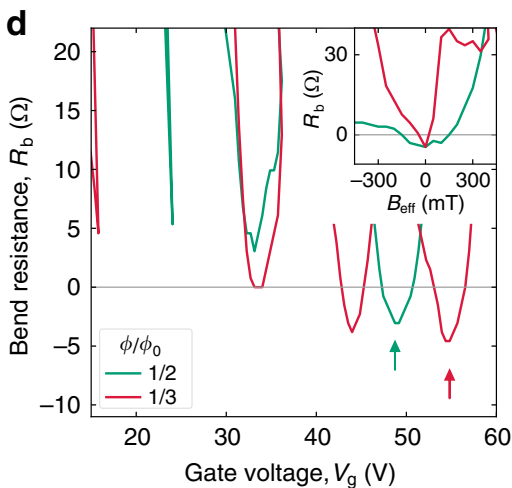

C

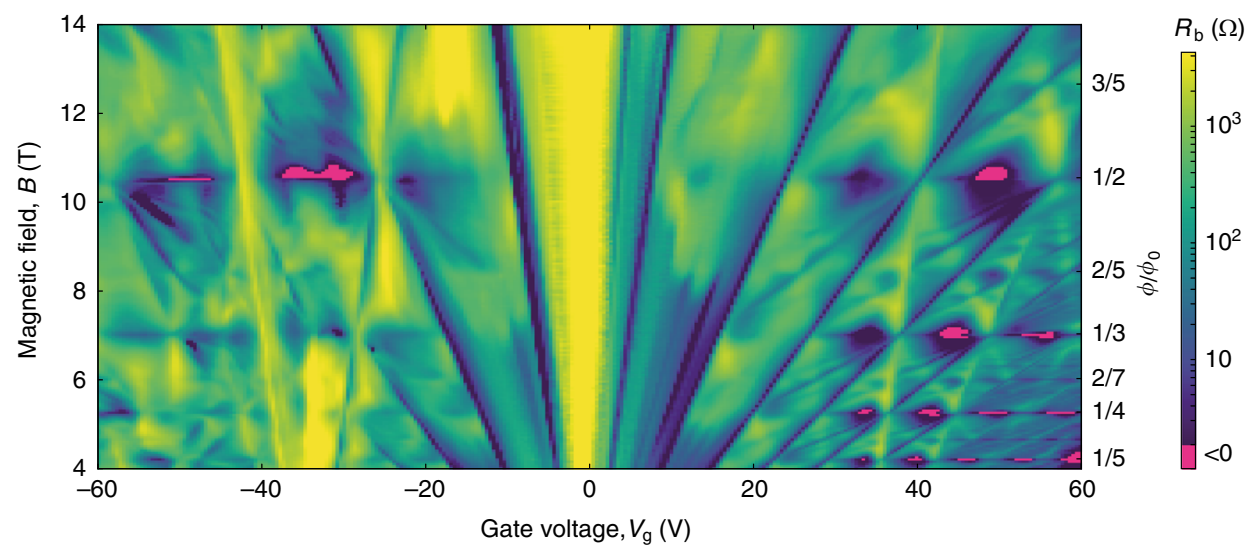

Fig. 2 Ballistic transport of BZ fermions over micrometer distances. a Schematic of bend resistance measurements. Current $/$ is applied between contacts 3 and 4 , and voltage $V_{\mathrm{b}}$ is measured between 2 and 1 , yielding the bend resistance, $R_{\mathrm{b}}=V_{\mathrm{b}} / l$. The voltage is positive for diffusive transport but becomes negative, if charge carriers move directly from current injecting contact 3 into voltage probe 1 (as shown by the red arrow). $\mathbf{b}$ Bend resistance for Dirac fermions in zero $B$ (device $\mathrm{D} 2$ with $W=4 \mu \mathrm{m}$ and $\theta \approx 0^{\circ}$ ). Inset: $R_{\mathrm{b}}(B)$ taken at the minimum indicated by the arrow in the main plot. $\mathbf{c}$ Map $R_{\mathrm{b}}\left(V_{\mathrm{g}}, B\right)$ for the same device. $B$ was changed in steps of $50 \mathrm{mT}$. Pockets of negative $R_{\mathrm{b}}$ appear along $\phi / \phi_{0}=1 / q$ and are seen in magenta. $\mathbf{d}$ Cross-sections from (c) for $q=$ 2 and 3. The inset shows sign reversals in $R_{\mathrm{b}}$ plotted as a function of $B_{\text {eff }}=B-B_{\mathrm{p} / \mathrm{q}}$ for the minima marked by the color-coded arrows. $T=2 \mathrm{~K}$ for all the plots.

$1 / 3$ and $1 / 4$ by $\Delta v=3$ and 4 , respectively (see Fig. $3 \mathrm{~b}$ ). Therefore, the observed degeneracies were equal to $q$. Note that the measured Hall conductance also exhibited quantized values in steps of $q e^{2} / h$ (Supplementary Note 7). Examining the Wannier diagram further, we find that $\Delta v$ was equal to $q$, independently of numerator $p$ and for all LLs stemming from the same $B_{\mathrm{p} / \mathrm{q}}$. This observation agrees with the theoretical expectation that there should be $q$ equivalent mini-valleys for each realization of $\mathrm{BZ}$ fermions ${ }^{18-27,30,31}$. The agreement takes into account that both spin and valley degeneracies for BZ fermions were lifted by exchange interactions ${ }^{14}$, as in the case of the main Dirac spectrum with its clearly lifted degeneracies (see the LLs marked in black in Fig. 3b). Therefore, the total degeneracy for BZ minibands in Fig. 3 was $g=4 q$. This is further supported by our measurements at a relatively high $T$ of $2 \mathrm{~K}$ that suppressed LLs with lifted spin and valley degeneracies (Supplementary Note 6). The same behavior (Landau mini-fans exhibiting the sequence $\Delta v=q$ ) was also observed in other parts of the Wannier diagram for both electron and hole doping (see, e.g., Fig. 4).

There is, however, one notable exception, which was observed for $\phi / \phi_{0}=1 / 3$ (left part of the Wannier diagram in Fig. 3b; dashed lines). In this case, LLs of BZ fermions with $q=3$ are separated only by $\Delta v=1$ so that all consecutive LLs from 3 to 12 are present on the fan diagram. The indicated minima in $\sigma_{\mathrm{xx}}$ were rather fragile, being rapidly smeared by $T$ or excitation current (Supplementary Note 7). Landau levels with $\Delta v=1$ at $\phi / \phi_{0}=1 / q$ do not exist within the single-particle Hofstadter-Wannier model ${ }^{16,17,21,22}$. Such 'extra' LLs have been observed previously and referred to as the fractional Bloch quantum Hall effect $(\mathrm{FBQHE})^{16}$ or symmetry-broken Chern insulator (SBCI) $)^{17}$. Theoretically, the many-body states can arise from interaction effects that lift the mini-valley degeneracy of BZ fermions. This may happen via mini-valley mixing due to the formation of charge-density waves (commensurate with the magnetic superlattice composed of $q$ unit cells of the underlying moiré pattern) or a Wigner crystal ${ }^{37}$ (states localized in a part of the magnetic supercell). Alternatively, the fully lifted degeneracy may occur through spontaneous mini-valley polarization of BZ LLs, analogous to spin/valley ferromagnetism in graphene ${ }^{14}$. In the latter case, the $\mathrm{BZ}$ fermion states localize in the momentum space around one of the mini-valleys but remain delocalized across the magnetic supercell.

\section{Discussion}

Some Landau mini-fans exhibited highly anomalous behavior at low $T$, which cannot be understood either within the HofstadterWannier model or by considering LLs of non-interacting BZ fermions and, to the best of our knowledge, was never reported before. From either perspective, individual LLs must evolve linearly in $V_{\mathrm{g}}$ and $B$, as indeed seen in most cases (e.g., Fig. 3). This is because the density of states on each BZ-fermion LL is proportional to $B_{\mathrm{eff}}=B-B_{\mathrm{p} / \mathrm{q}}$. Also, the long $(\sim 300 \mathrm{~nm})$ distance from graphene to the gate in our devices makes quantum capacitance corrections ${ }^{38,39}$ negligible, leading to the $n \propto V_{\mathrm{g}}$ dependence. Unexpectedly, we found that some Landau fans of BZ 

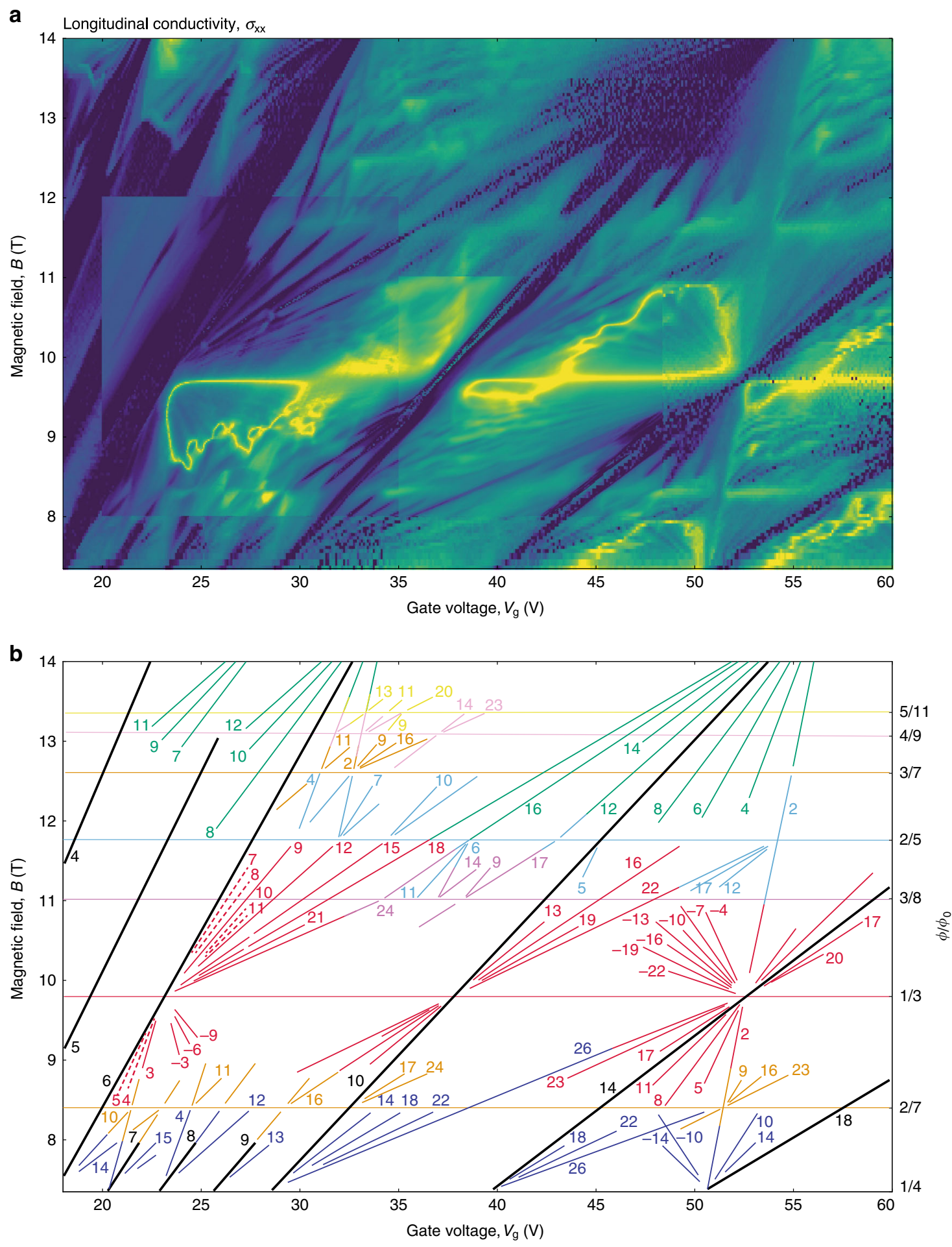

Fig. 3 Landau quantization in $\mathbf{B Z}$ minibands at $\mathbf{1 0} \mathbf{~} \mathbf{K K}$. a High-resolution map $\sigma_{\mathrm{xx}}\left(V_{\mathrm{g}}, B\right)$ for the electron-doped region indicated in Fig. 1c by the white rectangle (device D1). To better resolve LLs around $q=3, B$ was changed in steps of 10 to $20 \mathrm{mT}$ whereas data in other regions were acquired using 40 to $80 \mathrm{mT}$ steps. This has resulted in the contrast discontinuities seen in the map. Log color scale: indigo ( $230 \mathrm{nS}$ ) to yellow ( $7.8 \mathrm{mS}$ ) for, the entire map. b Minima from (a) are shown schematically. The color-coded numbers are the filling factors for the corresponding LLs. Thick black lines correspond to the main sequence of LLs for graphene's Dirac spectrum (spin and valley degeneracy lifted). The green, red, navy, blue, orange, magenta, pink, and yellow lines correspond to $q=2,3,4,5,7,8,9$, and 11 , respectively. Dashed red lines: minima due to lifted mini-valley degeneracy.

fermions exhibited bending and staircase-like features. Figure 4 shows examples of such behavior for the case of hole doping. Both bending and staircases appear away from NPs, in the regions close to BZ fermions' vHS. The observed bending towards the gate-voltage axis indicates that, in addition to the visible LLs, there can be some other electronic states that are populated in parallel. Because electron-electron interactions clearly play a significant role under the reported experimental conditions (e.g., they lift all the spectral degeneracies), it is reasonable to expect that interactions are also involved in the described anomalies. 
a

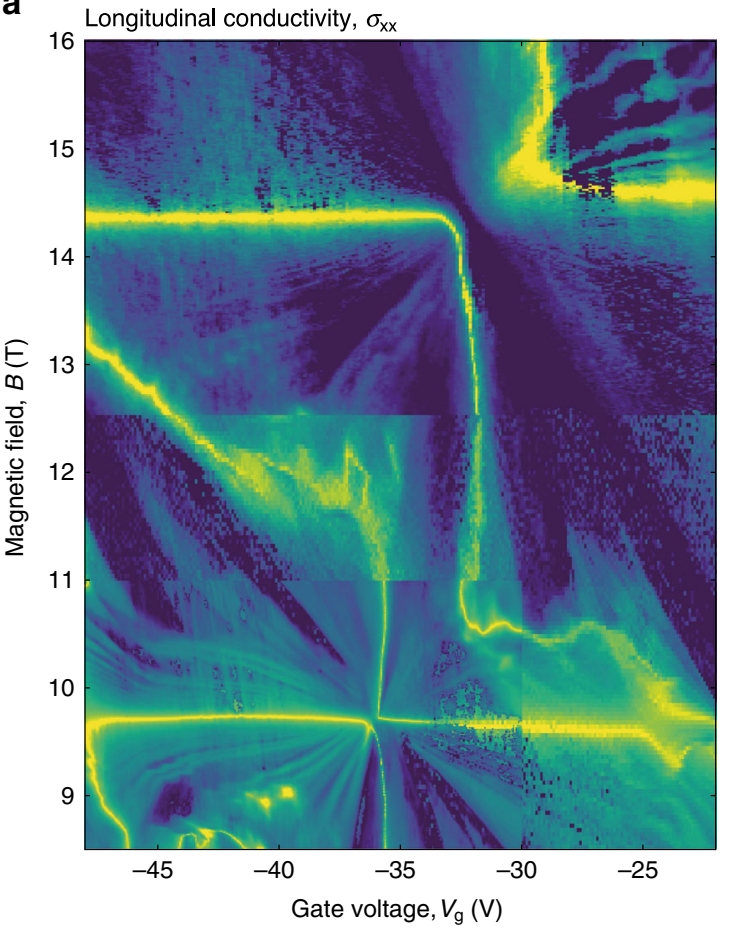

b

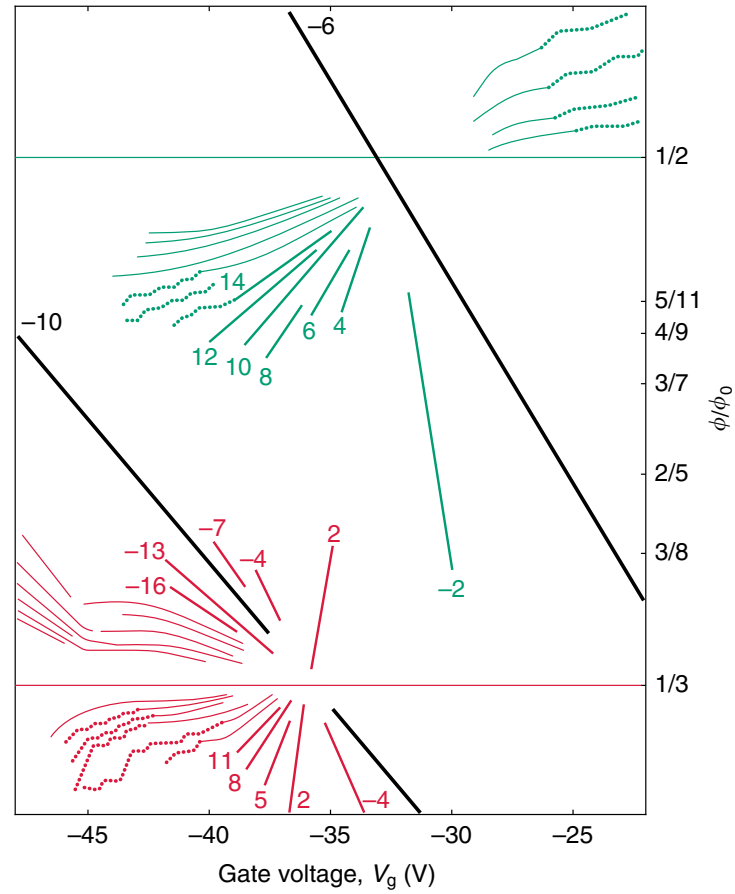

Fig. 4 Anomalous behavior of Landau levels in BZ minibands. a High-resolution $\sigma_{x x}\left(V_{g}, B\right)$ for the hole-doped region marked in Fig. 1c (device D1). The measurements below $B=12.5 \mathrm{~T}$ were carried out at $10 \mathrm{mK}$, and at $250 \mathrm{mK}$ in the fields above. $B$ was applied in steps of 16 and $40 \mathrm{mT}$ above and below $12.5 \mathrm{~T}$, respectively, except for the low left corner where the data were acquired at higher resolution of $10 \mathrm{mT}$ (seen as discontinuities in the contrast). Log scale: indigo $(310 \mathrm{nS})$ to yellow $(0.3 \mathrm{mS})$. b Schematics for the conductance minima found in (a). The same color coding as in Fig. $3 \mathrm{~b}$. The solid lines indicate LLs evolving as expected, linearly in $B$ and $V_{\mathrm{g}}$. The thin curves indicate the anomalous bending whereas the dotted curves show the staircase-like evolution observed for some LLs. All the anomalous features were highly reproducible and, for example, did not depend on the step size in $B$.

However, the usual suspects, such as negative compressibility cannot possibly explain our findings (in the latter case, LLs would bend toward the $B$ axis). The anomalous behavior is possibly due to an interplay of BZ-fermion LLs with quantized states originating from nearby vHS, which would lead to redistribution of charge carriers between states with the light and heavy effective masses. One possible scenario is localization of electrons within some parts of the magnetic supercell, because its size becomes notably larger than the magnetic length at $B>10 \mathrm{~T}$ (Wigner crystallization mentioned above). Unfortunately, we could not find any additional features that would enable us to decipher origins of the described anomalies and, therefore, have to leave them for further investigation.

\section{Methods}

Devices fabrication. The reported graphene-on-hBN superlattices were assembled using the standard dry transfer procedures and polydimethylsiloxane (PDMS) stamps coated with a polypropylcarbonate (PPC) layer ${ }^{7}$. The thicknesses of the used hBN crystals was between 20 and $70 \mathrm{~nm}$. After the trilayer stack was assembled, we used the standard electron beam lithography and reactive ion etching to define trenches for electrical contacts. $\mathrm{Cr} / \mathrm{Au}$ films were evaporated into the trenches. Finally, using ion etching again, the trilayer stack was shaped into a Hall bar mesa.

Electrical measurements. For electrical measurements, the standard lowfrequency lock-in technique was employed. At temperatures below $100 \mathrm{mK}$, we used excitation currents of $\sim 10 \mathrm{nA}$, and between 0.1 to $1 \mu \mathrm{A}$ at higher $T$. The carrier concentration was controlled by applying a DC gate voltage between graphene and the silicon substrate.

\section{Data availability}

The data that support the findings of this study are available from the corresponding author upon reasonable request.
Received: 23 June 2020; Accepted: 30 September 2020; Published online: 13 November 2020

\section{References}

1. Geim, A. K. \& Grigorieva, I. V. Van der Waals heterostructures. Nature 499, 419-425 (2013).

2. Novoselov, K. S., Mishchenko, A., Carvalho, A. \& Castro Neto, A. H. 2D materials and van der Waals heterostructures. Science 353, aac9439 (2016).

3. Yankowitz, M., Ma, Q., Jarillo-Herrero, P. \& LeRoy, B. J. van der Waals heterostructures combining graphene and hexagonal boron nitride. Nat. Rev. Phys. 1, 112-125 (2019).

4. Dean, C. R. et al. Boron nitride substrates for high-quality graphene electronics. Nat. Nanotech. 5, 722-726 (2010).

5. Mayorov, A. S. et al. Micrometer-scale ballistic transport in encapsulated graphene at room temperature. Nano Lett. 11, 2396-2399 (2011).

6. Wang, L. et al. One-dimensional electrical contact to a two-dimensional material. Science 342, 614-617 (2013).

7. Kumaravadivel, P. et al. Strong magnetophonon oscillations in extra-large graphene. Nat. Commun. 10, 3334 (2019).

8. Chen, S., Kraft, R., Danneau, R., Richter, K. \& Liu, M.-H. Electrostatic superlattices on scaled graphene lattices. Commun. Phys. 3, 71 (2020).

9. Wallbank, J. R., Patel, A. A., Mucha-Kruczyński, M., Geim, A. K. \& Fal'ko, V. I. Generic miniband structure of graphene on a hexagonal substrate. Phys. Rev. B 87, 245408 (2013).

10. Yankowitz, M. et al. Emergence of superlattice Dirac points in graphene on hexagonal boron nitride. Nat. Phys. 8, 382-386 (2012).

11. Ponomarenko, L. A. et al. Cloning of Dirac fermions in graphene superlattices. Nature 497, 594-597 (2013).

12. Dean, C. R. et al. Hofstadter's butterfly and the fractal quantum Hall effect in moiré superlattices. Nature 497, 598-602 (2013).

13. Hunt, B. et al. Massive Dirac fermions and Hofstadter butterfly in a van der Waals heterostructure. Science 340, 1427-1430 (2013). 
14. Yu, G. L. et al. Hierarchy of Hofstadter states and replica quantum Hall ferromagnetism in graphene superlattices. Nat. Phys. 10, 525-529 (2014).

15. Diez, M., Dahlhaus, J. P., Wimmer, M. \& Beenakker, C. W. J. Emergence of massless Dirac Fermions in Graphene's Hofstadter butterfly at switches of the quantum hall phase connectivity. Phys. Rev. Lett. 112, 196602 (2014).

16. Wang, L. et al. Evidence for a fractional fractal quantum Hall effect in graphene superlattices. Science 350, 1231-1234 (2015).

17. Spanton, E. M. et al. Observation of fractional Chern insulators in a van der Waals heterostructure. Science 360, 62-66 (2018).

18. Brown, E. Bloch electrons in a uniform magnetic field. Phys. Rev. 133, A1038-A1044 (1964).

19. Zak, J. Magnetic translation group. Phys. Rev. 134, A1602-A1606 (1964).

20. Azbel, M. Y. Energy spectrum of a conduction electron in a magnetic field. Sov. Phys. JETP 19, 634-645 (1964).

21. Wannier, G. H. A result not dependent on rationality for Bloch electrons in a magnetic field. Phys. Status Solidi 88, 757-765 (1978).

22. Hofstadter, D. R. Energy levels and wave functions of Bloch electrons in rational and irrational magnetic fields. Phys. Rev. B 14, 2239-2249 (1976).

23. Streda, P. Quantised Hall effect in a two-dimensional periodic potential. $J$. Phys. C. Solid State Phys. 15, L1299-L1303 (1982).

24. Rhim, J.-W. \& Park, K. Self-similar occurrence of massless Dirac particles in graphene under a magnetic field. Phys. Rev. B 86, 235411 (2012).

25. Rammal, R. Landau level spectrum of Bloch electrons in a honeycomb lattice. J. Phys. 46, 1345-1354 (1985).

26. Delplace, P. \& Montambaux, G. Semi-Dirac point in the Hofstadter spectrum. Phys. Rev. B 82, 035438 (2010).

27. Chen, X. et al. Dirac edges of fractal magnetic minibands in graphene with hexagonal moiré superlattices. Phys. Rev. B 89, 075401 (2014).

28. Krishna Kumar, R. et al. High-temperature quantum oscillations caused by recurring Bloch states in graphene superlattices. Science 357, 181-184 (2017).

29. Krishna Kumar, R. et al. High-order fractal states in graphene superlattices. Proc. Natl Acad. Sci. USA 115, 5135-5139 (2018).

30. Thouless, D. J., Kohmoto, M., Nightingale, M. P. \& den Nijs, M. Quantized Hall conductance in a two-dimensional periodic potential. Phys. Rev. Lett. 49, 405-408 (1982).

31. Landau, L. D. \& Lifschitz, E. M. Statistical Physics Part 2-The theory of the condensed state. in Course of Theoretical Physics (Pergamon Press Ltd., 1980).

32. Woods, C. R. et al. Commensurate-incommensurate transition in graphene on hexagonal boron nitride. Nat. Phys. 10, 451-456 (2014).

33. Wang, L. et al. New generation of moiré superlattices in doubly aligned hBN/ graphene/hBN heterostructures. Nano Lett. 19, 2371-2376 (2019).

34. Wang, Z. et al. Composite super-moiré lattices in double-aligned graphene heterostructures. Sci. Adv. 5, eaay8897 (2019).

35. Finney, N. R. et al. Tunable crystal symmetry in graphene-boron nitride heterostructures with coexisting moiré superlattices. Nat. Nanotech. 14, 1029-1034 (2019).

36. Beenakker, C. W. J. \& van Houten, H. Quantum transport in semiconductor nanostructures. Solid State Phys. - Adv. Res. Appl. 44, 1-228 (1991).

37. DaSilva, A. M., Jung, J. \& MacDonald, A. H. Fractional Hofstadter states in graphene on hexagonal boron nitride. Phys. Rev. Lett. 117, 036802 (2016).
38. Chen, Z. \& Appenzeller, J. Mobility extraction and quantum capacitance impact in high performance graphene field-effect transistor devices. In 2008 IEEE International Electron Devices Meeting 1-4 (IEEE, 2008).

39. $\mathrm{Yu}, \mathrm{G}$. L. et al. Interaction phenomena in graphene seen through quantum capacitance. Proc. Natl Acad. Sci. USA 110, 3282-3286 (2013).

\section{Acknowledgements}

This work was supported by the Engineering and Physical Sciences Research Council (EPSRC) Grand Challenges (EP/N010345), Lloyd's Register Foundation, Graphene Flagship, the Royal Society and the European Research Council (grant Vander). A.I.B. acknowledges support from the Graphene NOWNANO Doctoral Training Centre.

\section{Author contributions}

P.K., N.X., and M.H. fabricated devices. J.B., R.K.K., L.A.P., C.M., M.K., and A.I.B performed transport measurements with help from A.M, M.D.T., and J.R.P. J.B., P.K., R.K.K., L.A.P., V.I.F, A.K.G., and A.I.B. performed data analysis. T.T. and K.W. provided the hBN crystals. J.B., P.K., V.I.F., A.I.B., and A.K.G. wrote the paper. All authors contributed to the discussions.

\section{Competing interests}

The authors declare no competing interests.

\section{Additional information}

Supplementary information is available for this paper at https://doi.org/10.1038/s41467 020-19604-0.

Correspondence and requests for materials should be addressed to A.K.G. or A.I.B.

Peer review information Nature Communications thanks the anonymous reviewers for their contribution to the peer review of this work.

Reprints and permission information is available at http://www.nature.com/reprints

Publisher's note Springer Nature remains neutral with regard to jurisdictional claims in published maps and institutional affiliations.

(c) (i) Open Access This article is licensed under a Creative Commons Attribution 4.0 International License, which permits use, sharing, adaptation, distribution and reproduction in any medium or format, as long as you give appropriate credit to the original author(s) and the source, provide a link to the Creative Commons license, and indicate if changes were made. The images or other third party material in this article are included in the article's Creative Commons license, unless indicated otherwise in a credit line to the material. If material is not included in the article's Creative Commons license and your intended use is not permitted by statutory regulation or exceeds the permitted use, you will need to obtain permission directly from the copyright holder. To view a copy of this license, visit http://creativecommons.org/ licenses/by/4.0/.

(c) Crown 2020 